\title{
Sequence Homology Search Based on Database Indexing Using the Profile Hidden Markov Model
}

\author{
Qiang Xue*, James Cole ${ }^{\dagger}$, Sakti Pramanik* \\ * Department of Computer Science and Engineering ${ }^{\dagger}$ Department of Microbiology \\ Michigan State University, East Lansing, Michigan 48824, USA \\ Email: xueqiang@msu.edu,pramanik@cse.msu.edu,colej@msu.edu
}

\begin{abstract}
The Profile Hidden Markov Model (PHMM) has received increasing attention in the field of protein homology detection, since profile-based methods are much more sensitive in detecting distant homologous relationships than pairwise methods. Pure dynamic-programming-based systems are often used for PHMM searches. However, these dynamic-programmingbased systems are very time consuming for a large database. For instance, it may take approximately 15 minutes to search a short model of length 12 in the GenBank protein sequence database. Instead of searching the database sequentially, we search the database based on a tree-structured database indexing, called the HD-tree. The HD-tree is able to reduce the PHMM search time significantly without reducing the quality of search results. Performance of search using the HD-tree is compared with that of HMMER [1], a popular implementation of PHMM for protein sequence analysis. It is shown that the HD-tree approach is orders of magnitude faster than HMMER for short queries.
\end{abstract}

\section{INTRODUCTION}

Searching Genome sequence databases for homology is an important area of study. Because these databases are very large, query execution time is an important parameter that is considered for optimization. In traditional databases, indexes are normally used to speed up the search time. However, for sequence databases traditional indexing does not work because search criterion is based on edit distance. Current dynamic programming based approaches have high run-time complexity and for a large database the time can be quite high. BLAST, a popular sequence database search tool, addresses this problem using a two step approach. Unfortunately, for more complex searches involving multi sequence alignment, the time complexity grows faster with database size. In this research we have applied dynamic programming based search tools on a database index, minimizing the run time of the algorithm significantly. With this approach, the sequence database can be much larger than the available RAM memory, and the database can stay on disk.

\section{A. Profile Analysis}

Profile analysis has long been a useful method in finding and aligning distantly related sequences [2]. A profile is a description of the consensus (e.g., probability of a residue) of a multi-sequence alignment from a group or "family" of homologous sequences. It uses a position-specific scoring system to capture the information of conservation at various positions in a multi-sequence alignment. This makes it a much more sensitive method for searching genomic sequences than pairwise methods (e.g., BLAST or FASTA) that use a positionindependent scoring system.

\section{B. The Profile Hidden Markov Model}

Functional biological sequences typically come in families. Just as a pairwise alignment captures the relationship between two sequences, a multi-sequence alignment can show how the sequences in a family relate to each other. It is desirable to provide a consensus model for a multi-sequence alignment, so that the relationship between a new sequence and the family can be identified. In [3], a particular type of Hidden Markov Model (HMM) is introduced. This type of HMMs is well suited for representing profiles of multi-sequence alignments, and is called the Profile Hidden Markov Model (PHMM). Unlike the general HMMs, PHMMs are strongly linear, leftright models. There are three states at each consensus column of a multi-sequence alignment: "match", "insert", and "delete". A "match" state models the distribution of residues allowed in a column. An "insert" and "delete" state at each column models insertion and deletion of one or more residues between this column and the next, respectively. A small PHMM corresponding to a short multi-sequence alignment is shown in Figure 1. Algorithms for generating a PHMM from a multisequence alignment are described in [4].

Searching based on PHMM in a large sequence database is very time consuming. We have developed a database index based sequence searching called the HD-tree, to speed up the search time significantly.

\section{HD-TREE AND ITS BASIC STRUCTURE}

The HD-tree adopts a hybrid RAM/disk-based structure, in which leaf nodes are stored on the disk so that a large database can be indexed, and internal nodes are kept in the RAM to achieve greater efficiency. The HD-tree incorporates and extends some indexing strategies of the digital tree and the $\mathrm{B}^{*}$-tree [5], taking advantage of their strengths in search performance, compression capability, and storage utilization. The structure and construction algorithms of the HD-tree are presented in the following sections.

The HD-tree is an unbalanced and ordered tree (see the example in Figure 2). An internal node, $\delta$, of the HD-tree contains a list of pairs $L(\delta)=\left\{\left(a_{1}, P_{1}\right),\left(a_{2}, P_{2}\right), \ldots,\left(a_{m}, P_{m}\right)\right\}$, where $P_{i}$ is a pointer to its child node; $a_{i}(1 \leq i \leq m)$ is a letter from $\Lambda$, called the label of $P_{i}$; and $a_{1}<a_{2}<\ldots<a_{m}$, 


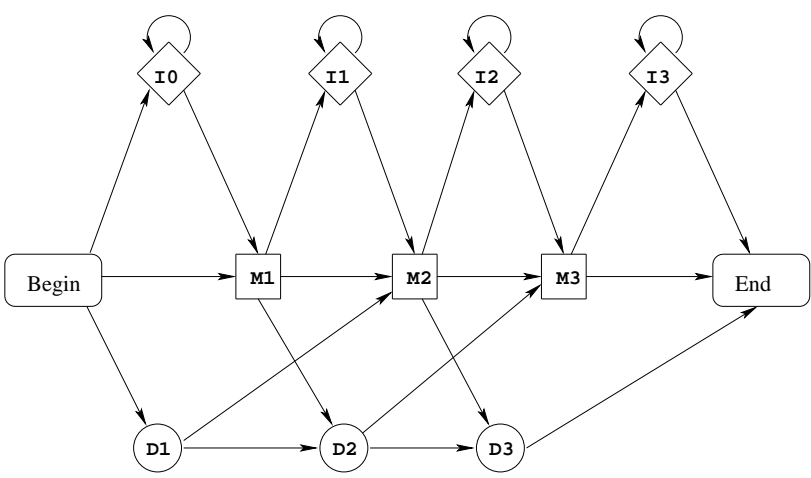

The Multi-Sequence

Alignment
$\begin{array}{lll}1 & 2 & 3 \\ C & A & 0\end{array}$
C A
$\begin{array}{lll}\text { C } & G & \text { E } \\ \text { C } & \text { A } & \text { Y }\end{array}$
C $A$ A
Emission Probability: M1: $\mathrm{C}=6 / 25$, other $=1 / 25$
M2: $A=4 / 25, G=2 / 25, K=2 / 25$, other $=1 / 25$ M3: $\mathrm{D}=3 / 25, \mathrm{E}=2 / 25, \mathrm{Y}=3 / 25$, other $=1 / 25$

Fig. 1. A small PHMM (right) representing a small multiple alignment of fi ve protein sequences (left) with three consensus columns. Squares represent match states (M1-M3). The 20 emission probabilities are calculated using Laplace's rule (i.e., each missing residue is counted one). Insert states (diamonds I0-I3) also have 20 emission probabilities (assume to be the same as the background distribution). Delete states (circles labeled D0-D3) are "mute" states that have no emission probabilities. State transition probabilities are shown as arrows.

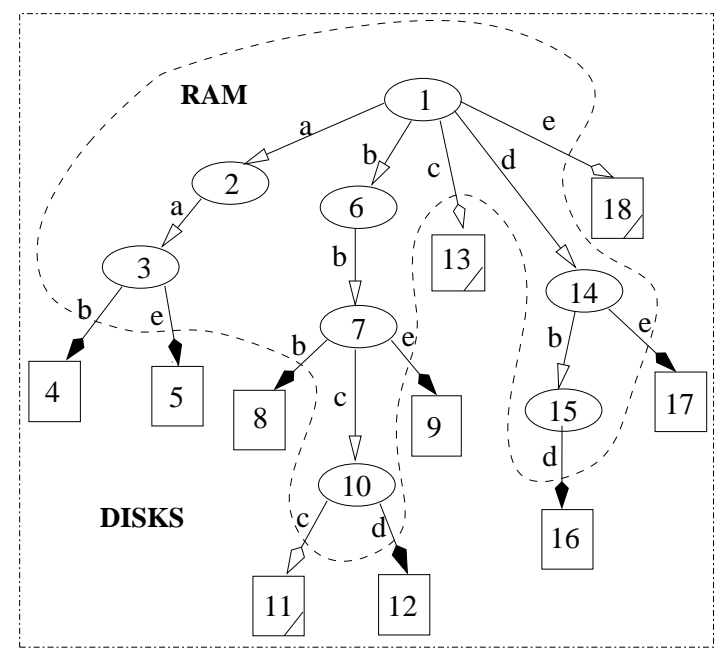

Fig. 2. An HD-tree

such that the pointers are ordered according to their labels. The order of siblings (the nodes who have the same parent) are determined by the pointers. For example, the left sibling of node 6 is node 2 , while the right sibling of node 6 is node 13 . Leaf nodes, which are implemented as disk blocks, contain the suffixes of indexed strings. The path string of a tree node is the concatenation of the labels along the path traversing from the root to the node. The path string of the root is empty. Since an HD-tree node can be uniquely identified by its path string, a path string is also called an id-string (i.e., identification string) of the corresponding node. Let $I D(\delta)$ denote the id-string of a tree node $\delta$. In Figure 2, ID $(2)=a, I D(9)=b b e$, and
$I D(15)=d b$.

An efficient sort/merge based algorithm to create an HD-tree is given in [6].

\section{A. HD-tree Search Algorithm}

Using suffix trees and suffix arrays to perform approximate string matching has been studied for years [7], [8], [9], [10]. Most of these research are focused on simple edit distance (also called $k$-difference problem), where a constant cost (e.g., unitary cost) is used for insertion, deletion, and substitution. Since genomic sequence searches use substitution matrices and affine gap cost model, algorithms based on simple edit distance cannot be directly applied.

The HD-tree index is created from the sequence database using overlapping words of length $L$. Therefore, it can be viewed as a suffix tree with maximum height of $L$. The algorithm of sequence search in the HD-tree is an extension of the dynamic programming algorithm applied to a suffix tree for approximate string matching based on the edit distance [9], [8], [11]. Substitution matrices and affine gap cost model are integrated in the algorithm. Imagine the need to obtain the best matching score, score $(\alpha, \beta)$, between sequences $\alpha=a_{1} \ldots a_{m}$ and $\beta=b_{1} \ldots b_{n}$. A table of $(m+1) \times(n+1)$ cells is created. Cell $C_{i, j}$ (i.e., $i$ th row and $j$ th column) contains the value $c_{i, j}$, which is the maximum achievable score by matching $a_{1} \ldots a_{i}$ and $b_{1} \ldots b_{j}$. The following equations define the calculation of $c_{i, j}$ :

$$
\begin{aligned}
& o=\text { the gap-open penalty (negative) } \\
& e=\text { the gap-extension penalty (negative) } \\
& m_{a, b}=\text { the substitution score for symbol } a, b \\
& \operatorname{gap}\left(C_{i, j}, C_{i^{\prime}, j^{\prime}}\right)=\left\{\begin{array}{l}
o \text { if from cell } C_{i, j} \text { to cell } C_{i^{\prime}, j^{\prime}} \\
\text { opens a gap } \\
e \text { if from cell } C_{i, j} \text { to cell } C_{i^{\prime}, j^{\prime}} \\
\text { extends a gap }
\end{array}\right. \\
& c_{0,0}=0 \\
& c_{i, 0}=o+e * i, \quad 0<i \leq m \\
& c_{0, j}=-\infty, \quad 0<j \leq n \\
& c_{i, j}=\max \left\{\begin{array}{l}
c_{i-1, j}+\operatorname{gap}\left(C_{i-1, j}, C_{i, j}\right), \\
c_{i, j-1}+\operatorname{gap}\left(C_{i, j-1}, C_{i, j}\right), \\
c_{i-1, j-1}+m_{a_{i}, b_{j}}
\end{array}\right.
\end{aligned}
$$

In the above equations, $c_{m, n}$ is the $\operatorname{score}(\alpha, \beta)$, and $c_{i, 0}$ is the score between $a_{1} \ldots a_{i}$ and empty string. $c_{0, j}$ is initialized to $-\infty$ so that gaps at the beginning of an overlapping word are not allowed. This is because the score of an alignment with beginning gaps is always less than that of the same alignment without the beginning gaps. Since the HD-tree indexes all overlapping words, the alignment with beginning gaps is a redundant result of the same alignment without the beginning gaps. Figure 3 shows a dynamic programming table for the HD-tree.

Recall that in the HD-tree, each tree node at level $x$ corresponds to an id-string, $a_{1} \ldots a_{x}$, which is the concatenation of the labels along the path from the root to the tree node. Since HD-tree indexes overlapping words, every potential match can be found by traversing the HD-tree from root to 


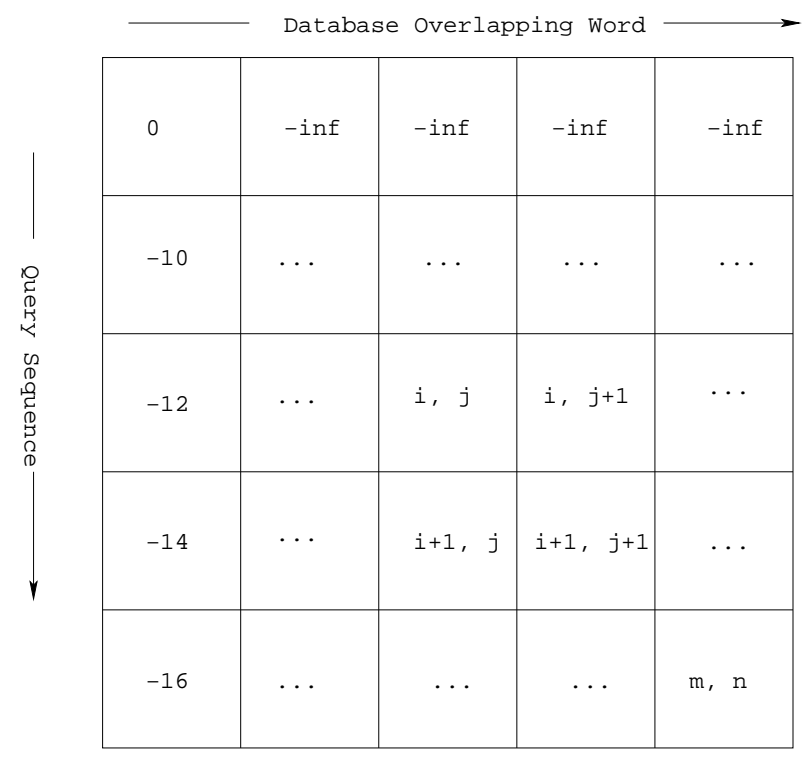

Gap-open penalty: $-10 \quad$ Gap-extension penalty: -2

Fig. 3. An Example of a Dynamic Programming Table Used in the HD-tree.

leaves. Therefore, query answers can be found by starting at the root and following every branch, until a match is found or the id-string of the current tree node cannot be the prefix of a possible match (i.e., any string under this sub-tree is not an answer, and the sub-tree is abandoned for the search).

Given a query string, $q=q_{1} \ldots q_{k}$, and a minimum matching score, $S$, an algorithm is designed to determine the matching score between $q$ and string $\alpha^{\prime}$, using the dynamic programming described in Equation 1. The details of the algorithm is given in [6]. In order to traverse the HD-tree and abandon a subtree that does not contain query answers, the algorithm must be able to meet the following criteria: (a) considering the string $\alpha^{\prime}$ incrementally, (b) determining when $\operatorname{score}\left(q, \alpha^{\prime}\right) \geq S$, and (c) determining when score $\left(q, \alpha^{\prime} \beta^{\prime}\right)<S$ for any string, $\beta^{\prime}$.

Assume a table of size $(k+1) \times(h+1)$ is created, where $k$ is the query length, and $h$ is the height of the HD-tree. Starting from the root, the algorithm descends recursively through every branch of the HD-tree. When descending through a branch labeled by letter $a$, the algorithm appends $a$ to current string, $\alpha^{\prime}$, and compute a table column corresponding to $a$ (see Figure 4). There are three possibilities for a given $\alpha^{\prime}$ :

(1) If the $\operatorname{score}\left(q, \alpha^{\prime}\right) \geq S$, all the leaves of the current sub-tree are reported as answers.

(2) If $\operatorname{score}\left(q, \alpha^{\prime} \beta^{\prime}\right)<S$ for any string, $\beta^{\prime}$, the sub-tree corresponding to $\alpha^{\prime}$ is abandoned immediately.

(3) Otherwise, the algorithm continues recursively descending through the HD-tree.

In order to fulfill criterion (3), the heuristic to determine whether score $\left(q, \alpha^{\prime} \beta^{\prime}\right)<S$ for any string, $\beta^{\prime}$, is as follows: assume the cell $C_{i,\left|\alpha^{\prime}\right|}$ contains the maximum score in column $\left|\alpha^{\prime}\right|$ (i.e., cells $C_{0,\left|\alpha^{\prime}\right|}$ to $\left.C_{k,\left|\alpha^{\prime}\right|}\right)$, then the maximum achievable score equals to $\operatorname{score}\left(q, \alpha^{\prime} q_{i+1} \ldots q_{k}\right)$ (i.e., the rest of matching is an exact match). If $\operatorname{score}\left(q, \alpha^{\prime} q_{i+1} \ldots q_{k}\right)<S$, then for any string, $\beta^{\prime}$, score $\left(q, \alpha^{\prime} \beta^{\prime}\right)<S$, where

$$
\begin{aligned}
& \operatorname{score}\left(q, \alpha^{\prime} q_{i+1} \ldots q_{k}\right) \\
& =\operatorname{score}\left(q_{1} \ldots q_{i}, \alpha^{\prime}\right)+\operatorname{score}\left(q_{i+1} \ldots q_{k}, q_{i+1} \ldots q_{k}\right) .
\end{aligned}
$$

An example of traversing the HD-tree and computing the dynamic programming table for a sequence search is shown in Figure 4. Compared with the standard dynamic programming method (which computes $n$ columns), the HD-tree significantly reduces the computation. For example, in Figure 4, only 10 columns are computed (this does include the columns computed within the shaded leaf node). This is because: (1) since the HD-tree indexes overlapping words, computing one table column at a tree node is equivalent to computing multiple table columns in a standard dynamic programming; (2) a subtree may be abandoned if it is impossible to satisfy the search criterion in the sub-tree.

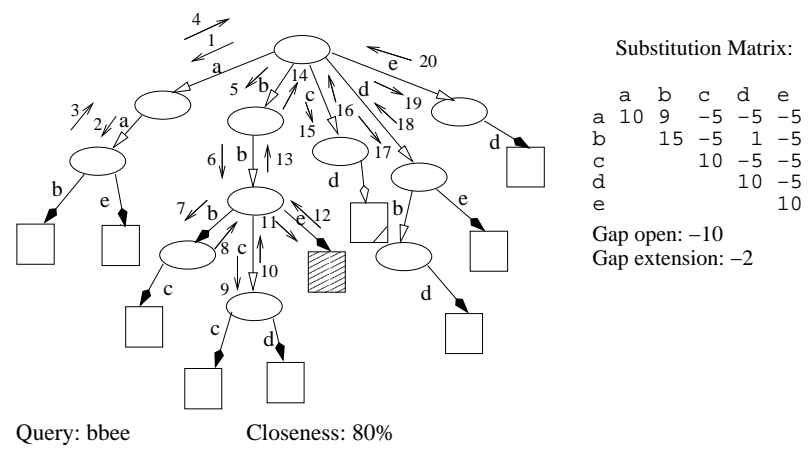

Maximum Score $=15+15+10+10=50$

Minimum Score $=50 * 80 \%=40$

Array msc $=\{35,20,10,0\}$

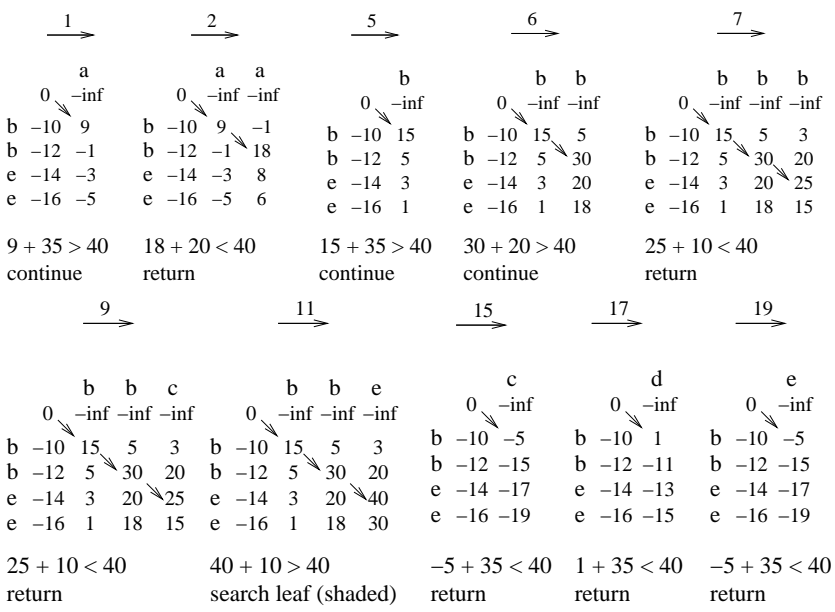

Fig. 4. An example of a genomic sequence search using the HD-tree. Arrow shows the traversing path. Each downward arrow computes one column (the right-most) of the table, and upward arrows do not compute matrix.

\section{SEARChing PHMM Using The HD-TREE}

The algorithm to search PHMM in the HD-tree is described in detail in [6]. Assume a query model, $q=q_{1 \ldots k}$, and a minimum matching score, $S$. Starting from the root, the algorithm descends recursively through every branch of the 
HD-tree. When descending through a branch labeled by the letter, $a$, the algorithm adds $a$ to the current string, $\alpha^{\prime}$. Assume $\operatorname{phmm}\left(q, \alpha^{\prime}\right)$ is the highest achievable score by matching $q$ and $\alpha^{\prime}$. There are three possibilities for the given $\alpha^{\prime}$ :

(1) If the $\operatorname{phmm}\left(q, \alpha^{\prime}\right) \geq S$, all the leaves of the current sub-tree are reported as answers.

(2) If $p h m m\left(q, \alpha^{\prime} \beta^{\prime}\right)<S$ for any string, $\beta^{\prime}$, the branch is abandoned immediately.

(3) Otherwise, the algorithm continues recursively descending through the HD-tree.

The score of the best path to match the sub-sequence, $\alpha^{\prime}=$ $a_{1 \ldots i}$, and the query model, $q=q_{1} \ldots q_{k}$, is computed using the Viterbi Equation.

\section{THE HMMER PACKAGE}

HMMER is a freely distributable package (current version is 2.3.2) for protein sequence analysis using PHMM [1]. It contains a set of programs useful for building and searching PHMMs. HMMER is hosted at Washington University in St. Louis, and is one of the most popular packages used by biologists to detect distant homologous relationships using PHMMs. HMMER is used to search for sequences that belong to a known protein family constructed from a multi-sequence alignment. The protein family, like most protein families, is so diverse that a BLAST search may fail to report even the known members in the family. HMMER is also used in automated annotation of the domain structure of proteins, and automated construction and maintenance of large multisequence alignment databases [12].

As shown in [12], HMMER uses 'Plan 7" model architecture, which contains 7 transitions per node in the main model. 'Plan 7' has five special states: ' $\mathrm{S}$ ', 'N', 'C', 'T', and ' $\mathrm{J}$ '. When combined with entry probabilities from ' $\mathrm{B}$ ' state and exit probabilities to ' $E$ ' state, these special states control unique features of the model. For instance, how likely the model is to generate various sorts of local or multi-hit alignments.

In traditional pairwise alignments, distinction is made between global Needleman-Wunsch and local Smith-Waterman algorithms. However, in the Plan 7 architecture, local versus global alignment in HMMER is controlled by transition probabilities. For example, local alignments with respect to the model are achieved by non-zero state transition probabilities from the begin state, 'B', to internal match states, and from internal match states to the end state, 'E'. Local alignments with respect to the sequence are achieved by non-zero state transitions on the flanking insert states, ' $\mathrm{N}$ ' and ' $\mathrm{C}$ '. More than one hit to the PHMM per sequence is achieved by a cycle of non-zero transitions through the special insert state, ' $J$ '. The HD-tree adopts the Plan 7 architecture of the HMMER package, so that local, global, and multi-hit alignments can be easily controlled by transition probabilities in PHMMs. Using the same architecture also allows better comparison between HMMER and the HD-tree. To implement the Plan 7 architecture into HD-tree we modified our HD-tree search algorithm to incorporate extra states. The details of the algorithm is given in [6].

\section{PERFORMANCE COMPARISONS}

In this section, the performance of HD-tree approach is compared with that of the HMMER for sequence searching using PHMMs [1]. The entire GenBank protein database is used as the sample database. The HD-tree is created from the entire GenBank Protein Database using overlapping words of length 20. Experiments are conducted on a Linux PC with 512MB RAM and $1.8 \mathrm{GHz}$ Pentium 4 processor. Queries are generated from the popular PFAM database. Both synthetic and real queries are used in the experiments. We use E-value and the closeness for search criterion.

\section{A. Queries based on PFAM}

PFAM (Protein FAMilies) is a large collection of multisequence alignments and PHMMs, covering many common protein families [13]. Genome projects, including both the human and fly, have used PFAM extensively for large scale functional annotation of genomic data [14]. PFAM version 18.0 (August 2005) is used in the experiments. It contains alignments and models for 7973 protein families, based on the Swiss-Prot 47.0 and SP-TrEMBL 30.0 protein sequence databases [15].

\section{B. Synthetic Queries}

The first set of experiments is conducted using synthetic queries, which are generated from long PHMMs in PFAM. The procedure of generating the synthetic queries is as follows. Assume a long PHMM, $M_{r}$, of length $L$. Transition and emission probabilities related to match, insert, and delete states of a synthetic query, $M_{s}$, of length $l$ are copied from the $i$ th to $(i+l)$ th state of $M_{r}$. Transition probabilities from 'B' state to any state in $M_{s}$ are copied from the first $l$ states of $M_{r}$, with transition probabilities from any state to ' $\mathrm{E}$ ' state in $M_{s}$ are copied from the last $l$ states of $M_{r}$. These synthetic queries are then run through a program, hmmcalibrate, provided by HMMER package. hmmcalibrate takes a PHMM and empirically determines parameters that are used to make searches more sensitive by calculating more accurate E-values.

In HD-tree edit distance based search is done directly unlike as in BLAST where the first phase is a filtering phase based on exact (or Hamming Distance) match. Therefore, all results are found in HD-tree based search as long as the search criterion (e.g., the E-value) is satisfied. Experiments have shown that the HD-tree finds all the results as are found by HMMER. Therefore, the quality of query results of the HD-tree is the same as that of HMMER. Table I shows the average query time with respect to query lengths and E-values using the HD-tree and HMMER. For the HD-tree, the results are the averages of 100 synthetic queries unless stated otherwise. However, since HMMER is very slow (10-15 minutes per query), only 10 synthetic queries are used for HMMER to generate the 
TABLE I

HD-TREE VERSUS HMMER, E-VALUE $=10$

\begin{tabular}{l|llllll}
\hline Qlen & 7 & 8 & 9 & 10 & 11 & 12 \\
\hline HMMER & 605.1 & 723.1 & 672.0 & 741.6 & 859.4 & 878.9 \\
HD-tree & 0.007 & 0.012 & 0.927 & 5.631 & 38.760 & 154.970 \\
\hline & \multicolumn{7}{c}{ Table value: Query time in seconds. }
\end{tabular}

average. It is seen from table 1 that the HD-tree is orders of magnitude faster than HMMER.

Besides the E-value, the HD-tree also uses closeness to evaluate the degree of similarity between two sequences. Evalue and closeness are exchangeable for a given query. Table II shows the relationship between closeness and E-value for the synthetic queries. It is shown that for a query of length 7 , even a near-exact match (i.e., closeness $=98 \%$ ) may result in E-value of at least 10 . This is one reason why short queries are executed very fast using the HD-tree.

TABLE II

Closeness versus E-VAlue

\begin{tabular}{l|llll}
\hline Qlen & Eval & AvgC & Eval & AvgC \\
\hline 7 & 10 & 98.0 & 100 & 93.8 \\
8 & 10 & 96.4 & 100 & 86.0 \\
9 & 10 & 90.6 & 100 & 75.1 \\
10 & 10 & 82.8 & 100 & 65.0 \\
11 & 10 & 72.8 & 100 & 55.2 \\
12 & 10 & 63.2 & 100 & 47.5 \\
\hline Qlen & Closeness & AvgEval & Closeness & AvgEval \\
7 & 60 & 3019.0 & 90 & 674.7 \\
8 & 60 & 1348.0 & 90 & 209.2 \\
9 & 60 & 628.6 & 90 & 69.8 \\
10 & 60 & 295.5 & 90 & 21.5 \\
11 & 60 & 134.5 & 90 & 5.8 \\
12 & 60 & 64.8 & 90 & 1.8 \\
\hline
\end{tabular}

Qlen: Query Length; Eval: E-value; AvgEval: Average E-value; $\operatorname{Avg} C$ : Average Closeness.

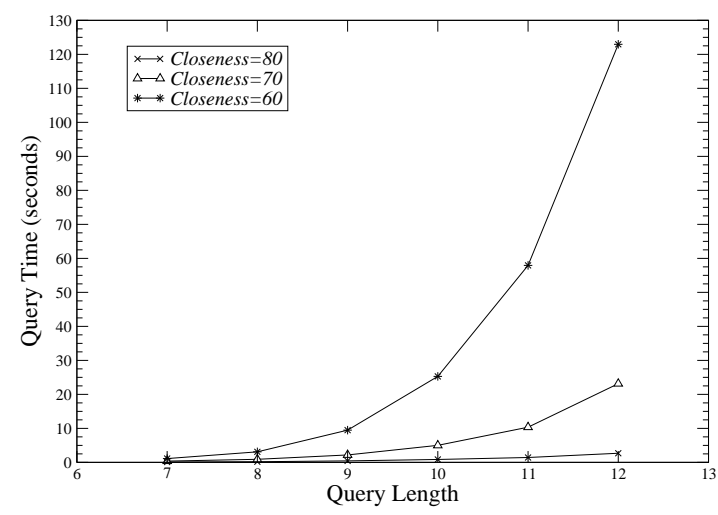

Fig. 5. HD-tree query time for synthetic PHMMs

Figure 5 illustrates the relationships between query time and query length for a given closenesses. It is shown that the query time decreases as the query length decreases or closeness increases. The relationship between query time and disk accesses, for queries of length 10, is shown in Table III. It is shown that the query time is closely related to the number of disk accesses. The percentage of accessed leaf nodes is very low, indicating good pruning power (i.e., the ability to abandon a sub-tree in a search) of the HD-tree based searches. Table IV presents the number of dynamic programming columns computed in sequence searches using the HD-tree, where "Ratio" is the number of columns computed by the HDtree divided by the number of columns computed by regular dynamic programming. This again shows a significant pruning power of HD-tree based searches on the number of columns to be computed in dynamic programming.

TABLE III

HD-TREE QUERY TIME FOR SYNTHETIC PHMMS; QUERY LENGTH $=10$

\begin{tabular}{lllll}
\hline $\begin{array}{l}\text { Closeness } \\
(\%)\end{array}$ & $\begin{array}{l}\text { Qtime } \\
\text { (seconds) }\end{array}$ & DiskAcc & $\begin{array}{l}\text { AccPerc } \\
(\%)\end{array}$ & AvgE-value \\
\hline 90 & 0.14 & 48 & 0.001 & 21.46 \\
80 & 0.88 & 299 & 0.011 & 51.77 \\
70 & 5.04 & 1989 & 0.072 & 125.4 \\
60 & 25.29 & 11153 & 0.407 & 295.5 \\
50 & 100.43 & 57781 & 2.107 & 684.8 \\
\hline
\end{tabular}

Qtime: query time; DiskAcc: the number of disk accesses; AccPerc: the percentage of accessed leaves.

TABLE IV

COMPUTATION OF DYNAMIC PROGRAMMING TABLE COLUMNS USING SYNTHETIC PHMMS, CLOSENESS $=60 \%$

\begin{tabular}{lll}
\hline PHMM Length & Column Computed & Ratio \\
\hline 7 & 10103 & $1.52844 \mathrm{E}-05$ \\
8 & 35335 & $5.34569 \mathrm{E}-05$ \\
9 & 117850 & 0.000177882 \\
10 & 348271 & 0.000526885 \\
11 & 899827 & 0.001361312 \\
12 & 2573057 & 0.003892673 \\
\hline
\end{tabular}

\section{Justification of Improved Query Time}

HMMER is a pure dynamic-programming-based method. It sequentially reads all database sequences, and compute one Dynamic Programming Table (DPT) column for each residue. For any query, reading the database takes approximately 40 seconds. However, the computation of DPT (approximately 661 million columns) dominates the query time. Therefore, the query time is relatively consistent, except some variations due to the post-processing of query results. The computational complexity of HMMER is $O(m n)$, where $m$ is the query length and $n$ is the database size.

On the other hand, as an index-based method, HD-tree significantly reduces DPT computation. This is due to the following two reasons. First, since the HD-tree indexes all overlapping words using a trie structure, it avoids repeated DPT computations for the subsequences (at different positions) having the same prefix. Second, during the tree traversal, a sub-tree may be abandoned (i.e., pruned) if a possible match is not possible within the sub-tree. For example, for query length of 7, the DPT columns computed by the HD-tree is only 101 , which is negligible compared to 661 million columns computed by HMMER. As the query length increases, the computation of DPT increases. However, even when the query length is 12 , the DPT columns computed by the HD-tree is still 
less than $0.1 \%$ of the DPT columns computed by HMMER. Therefore, the CPU time of the HD-tree is not the major factor of query performance. However, since the HD-tree stores leaf nodes on disks, the query time is dominated by the disk access time, which is proportional to the number of disk accesses (i.e., leaf-node accesses). Since the internal nodes of the HDtree corresponds to a trie, each tree node represents a string (i.e., a prefix of the overlapping words). A sub-tree can be pruned (i.e., do not access the leaf nodes in this subtree) if the best alignment between the query and the prefix string representing the sub-tree has exceded the maximum error (i.e., the maximum score minus the minimum score).

\section{Real Queries}

In order to compare the HD-tree with HMMER using real PHMMs, experiments are conducted on PHMMs obtained from the PFAM database directly. Table $\mathrm{V}$ shows the query time of the HD-tree and HMMER using these real PHMMs, where "Seq" is a given number to distinguish different queries having the same length. Although the overall performance trend is similar to that in synthetic queries, it is observed that the running time varies among queries having the same length. This is due to the fact that the HD-tree uses the prefix of a query to reduce the search space and the composition of a query affects the prefixes of the HD-tree. For example, if the first few states in a PHMM has more pruning power, the HDtree tends to be faster.

\section{CONCLUSION}

Database indexing based homology search using Profile Hideen Markov Model is presented. The results show a significant performance gain of the proposed approach over HMMER in PHMM search for short queries. As genomic sequence databases continue to grow, the benefit of using an index-based approach, such as the HD-tree, is more and more appealing than the linear-scan-based approach, such as HMMER and BLAST. The proposed approach is especially significant for complex sequence analysis tasks such as PHMM searches. We are currently developing extensions of HD-tree based approach for efficiently searching long queries.

\section{REFERENCES}

[1] 'Hmmer: profi le hmms for protein sequence analysis," October 27, 2005, http://hmmer.wustl.edu/.

[2] M. Gribskov, A. D. McLachlan, and D. Eisenberg, "Profi le analysis: Detection of distantly related proteins," vol. 84, 1987, pp. 4355-4358.

[3] A. Krogh, M. Brown, I. S. Mian, K. Sjolander, and D. Haussler, "Hidden markov models in computational biology: Applications to protein modeling," Journal of Molecular Biology, vol. 235, no. 15011531, 1994.

[4] R. Durbin, S. Eddy, A. Krogh, and G. Mitchison, Biological sequence analysis: Probabilistic models of proteins and nucleic acids. Cambridge University Press, 1998.

[5] D. Comer, 'Ubiquitous b-tree," ACM Comput. Surv., vol. 11, no. 2, pp. 121-137, 1979.

[6] Q. Xue, "The hybrid digital tree and its application to genomic sequence databases," Ph.D. dissertation, Michigan State University, East Lansing, USA, 2005.

[7] E. Ukkonen, "Approximate string-matching over suffi x trees," in Proceedings of the 4th Annual Symposium on Combinatorial Pattern Matching. Springer-Verlag, 1993, pp. 228-242.
TABLE V

QUERY TIME FOR REAL PHMMS; E-VALUE $=10$

\begin{tabular}{lllll}
\hline Qlen & Seq & $\begin{array}{l}\text { HD-tree } \\
\text { (seconds) }\end{array}$ & Closeness & $\begin{array}{l}\text { HMMER } \\
\text { (seconds) }\end{array}$ \\
\hline 7 & 1 & 0.386 & 74 & 563 \\
8 & 1 & 0.028 & 79 & 741 \\
8 & 2 & 0.363 & 67 & 704 \\
8 & 3 & 0.036 & 99 & 951 \\
8 & 4 & 0.002 & 99 & 741 \\
8 & 5 & 0.128 & 72 & 709 \\
9 & 1 & 4.661 & 57 & 660 \\
9 & 2 & 0.001 & 99 & 692 \\
9 & 3 & 4.871 & 58 & 671 \\
9 & 4 & 0.001 & 99 & 757 \\
10 & 1 & 0.168 & 81 & 740 \\
10 & 2 & 8.909 & 53 & 731 \\
10 & 3 & 0.612 & 76 & 724 \\
10 & 4 & 9.464 & 61 & 951 \\
10 & 5 & 3.168 & 70 & 728 \\
10 & 6 & 0.001 & 99 & 717 \\
11 & 1 & 5.685 & 47 & 809 \\
11 & 2 & 0.312 & 81 & 856 \\
11 & 3 & 5.312 & 66 & 821 \\
11 & 4 & 7.000 & 64 & 786 \\
11 & 5 & 1.112 & 66 & 844 \\
12 & 1 & 36.252 & 45 & 898 \\
12 & 2 & 160.322 & 48 & 875 \\
12 & 3 & 52.374 & 53 & 905 \\
12 & 4 & 229.911 & 41 & 843 \\
12 & 5 & 211.286 & 34 & 892 \\
\hline len: query length; Seq: query sequence number.
\end{tabular}

[8] _ , "On-line construction of suffi x-trees," Algorithmica, vol. 14, pp. 249-260, 1995 .

[9] D. Gusfi eld, Algorithms on Strings, Trees, and Sequences: Computer Science and Computational Biology. Cambridge: Cambridge University Press, 1997.

[10] G. Navarro and R. Baeza-Yates, "A hybrid indexing method for approximate string matching," Journal of Discrete Algorithms (JDA), vol. 1, no. 1, pp. 205-239, 2000, special issue on Matching Patterns.

[11] E. Hunt, M. P. Atkinson, and R. W. Irving, 'Database indexing for large dna and protein sequence collections," The VLDB Journal, vol. 11, no. 3 , pp. 256-271, 2002.

[12] S. R. Eddy, HMMER Userś Guide, October 2003 , $\mathrm{ftp} / / / \mathrm{ftp}$.genetics.wustl.edu/pub/eddy/hmmer/CURRENT/Userguide.pdf.

[13] A. Bateman, L. Coin, R. Durbin, R. D. Finn, V. Hollich, S. Griffi thsJones, A. Khanna, M. Marshall, S. Moxon, E. L. L. Sonnhammer, D. J. Studholme, C. Yeats, and S. R. Eddy, "The pfam protein families database," Nucleic Acids Res., vol. 32, pp. D138-D141, 2004.

[14] A. Bateman, E. Birney, L. Cerruti, R. Durbin, L. Etwiller, S. R. Eddy, S. Griffi ths-Jones, K. L. Howe, M. Marshall, and E. L. L. Sonnhammer, "The pfam protein families database," Nucleic Acids Res., vol. 30, no. 1, p. $276280,2002$.

[15] "The pfam database of protein families and hmms," August, 2005, http://pfam.wustl.edu/. 